\title{
Mucosal Route of Administration
}

National Cancer Institute

\section{Source}

National Cancer Institute. Mucosal Route of Administration. NCI Thesaurus. Code C38283.

Administration of a substance to the mucosa; often resulting in systemic action due to absorption. 